\title{
Impact of the gut microbiota on chemical risk assessment
}

\author{
A Wallace Hayes, \\ University of South Florida, \\ Tampa, FL USA
}

\begin{abstract}
The multitude of microbes that reside in the human intestine plays an important role in the health and well being of humans. Not only do chemicals affect the composition of the gut microbiota but they affect the uptake and metabolism of ingested chemicals in multiple ways. The interplay among gut microbes, ingested xenobiotics, and toxicological effects is complex and not well understood. Recent studies have shown that the gut microbiota is a key player in the toxicity of drugs and chemicals, which is typically overlooked in current approaches for risk assessment. The microbial community in the gut may mediate or mitigate detrimental effects of chemicals by various mechanisms. Hence, there is a need to include, or at least
\end{abstract}

consider, the gut microbiota as part of a human toxicological risk assessment protocol. Factors such as animal provider, batch/litter differences, and co-caging, among other factors, may significantly influence the outcome of a toxicity evaluation that is based on rodent experiments. A framework for assessment of gut microbiota upon exposure to drugs or chemicals is suggested. 